УДК 004.932.2

\title{
РАЗРАБОТКА АЛГОРИТМОВ АНАЛИЗА ИЗОБРАЖЕНИЙ ВУЛКАНОВ ДЛЯ МОНИТОРИНГА ОПАСНЫХ ПРИРОДНЫХ ПРОЦЕССОВ И ЯВЛЕНИЙ
}

\author{
Урманов Игорь Павлович1, \\ urmanov@ccfebras.ru \\ Сорокин Алексей Анатольевич1, \\ alsor@febras.net \\ Королев Сергей Павлович 1 , \\ serejk@febras.net
Мальковский Сергей Иванович1, sergey.malkovsky@gmail.com \\ 1 Вычислительный центр Дальневосточного отделения Российской академии наук, \\ Россия, 680000, Хабаровск, ул. Ким Ю Чена, 65.
}

\begin{abstract}
Актуальность. Системы видеонаблюдения являются важнейшим видом инструментальных средств наблюдений за состоянием вулканов. Высокая интенсивность сбора данных способствует формированию огромного массива изображений. При этом климатические особенности мест нахождения исследуемых объектов, а также не всегда стабильная работа каналов передачи данных являются причиной появления большого числа испорченных или неинформативных снимков. На их просмотр и исключение из архива затрачивается дополнительное время, что в иелом может сказаться на оперативности принятия решений, связанных с информированием заинтересованных лиц о возникновении опасных природных явлений. $B$ связи с этим актуальной является задача разработки методов и технологий анализа и фильтрации изображений для создания эфффективной системы видеонаблюдения за вулканами.

Цель: разработка алгоритмов для анализа изображений вулканов.

Методы: детектор грании Саппу, преобразование дискретных контуров в параметрические в виде ломанных линий, вычисление эталонных характеристик и сравнение с ними полученных данных, анализ частотных характеристик снимка с использованием преобразования Хаара, разложение изображения в многомаситабную пирамиду, составление вектора характерных визуальных признаков объекта, обнаружение искомого объекта с использованием SVM-классификатора.

Результаты. Алгоритмы анализа фотоснимков для оценки видимости вулканов и поиска яркостных аномалий.

Выводы. В рамках проведенного исследования авторами были разработаны алгоритмы для анализа изображений вулканов. Предложенные методы и подходы позволяют эфрфективно решать задачи оценки видимости объекта и детектирования яркостных аномалий. Созданные программные средства предоставляют набор инструментов для фрильтрации и экспертной оценки изображений с целью поиска возможных признаков активности вулкана. Полученные результаты могут быть использованы в работе систем мониторинга вулканической активности для обеспечения безопасности авиации и защиты населения.
\end{abstract}

\section{Ключевые слова:}

Изображения вулканов, вулканическая активность, яркостная аномалия, алгоритм, машинное обучение, программа.

\section{Введение}

Одним из эффективных средств наблюдений за опасными природными объектами являются системы видеонаблюдения. Как правило, в их состав входят стационарные видеокамеры и программно-аппаратные комплексы, обеспечивающие сбор, хранение и, в зависимости от назначения системы, специализированную обработку данных.

Сотрудниками Вычислительного центра ДВО РАН и Института вулканологии и сейсмологии ДВО РАН создана система видеонаблюдения, предназначенная для оперативного мониторинга состояния вулканов Камчатки [3]. В системе используются два типа видеокамер: работающие только в видимом диапазоне света (StartDot NetCam XL), и камеры с IR-cut фильтром, позволяющим вести съемку в темное время суток в чёрно-белом режиме (Axis P1343). На качество и информативность получаемых фотоснимков влияют многие факторы. Например, наличие тумана, облачности или осадков, засветки от солнечных лучей, а также перебои в работе видеооборудования и каналов передачи данных, что часто приводит к появлению в архиве испорченных изображений. В итоге вулкан может быть не виден на фотоснимке, а оценка его состояния затруднена или невозможна. С учетом высокой интенсивности наполнения архива системы и его большого объема для эффективной работы с ним требуется создание эффективных инструментов, обеспечивающих фильтрацию неинформативных изображений и поиск снимков с признаками активности вулканов.

Существующие в мире компьютерные системы для анализа и обработки фотоснимков вулканов, в первую очередь, ориентированы на работу с данными, полученными с термальных камер [4-6]. В работах $[7,8]$ представлены алгоритмы анализа снимков, сделанных в видимом диапазоне, но с заведомо четкой видимостью объекта. С их помощью на изображениях ведется поиск участков, яркость которых выше определенного порога $[9,10]$. Однако такие участки могут соответствовать и посторонним освещенным объектам. Одним из современных методов и подходов к 
решению задач классификации изображений является использование нейронных сетей, однако они требуют предварительного обучения на размеченных наборах снимков, объем которых может достигать сотен или тысяч единиц. Создание таких наборов является трудоемкой и продолжительной по времени задачей и требует разработки отдельных компьютерных средств и решений.

В настоящей работе рассматриваются алгоритмы и созданная на их основе компьютерная система, позволяющие проводить автоматизированную классификацию снимков, сделанных в светлое время суток (далее - дневные снимки), по степени видимости вулкана на них, а также обнаружение яркостных аномалий на снимках, сделанных в темное время суток с помощью камер с инфракрасным фильтром (далее ночные снимки). Дается описание разработанных методов и алгоритмов анализа данных, а также результаты их апробации на примере серий фотоснимков отдельных вулканов Камчатки.

\section{Алгоритм определения видимости вулкана на дневных снимках}

Определение видимости объекта на цифровом изображении можно рассматривать в контексте таких задач компьютерного зрения, как определение атмосферной видимости $[11,12]$ и детектирование изменений состояния объекта $[13,14]$. При этом методы и подходы, предлагаемые в указанных работах, не могут быть непосредственно использованы для определения видимости вулканов на снимках ввиду высокой чувствительности к неоднородности атмосферы на больших расстояниях и к изменчивости внешнего вида вулкана в зависимости от освещения и времени года. Поиск особенностей, свойственных вулкану, напротив, позволяет оценить видимость вулкана в самых различных условиях и ситуациях. В работе [15] в качестве таких особенностей предлагается использовать пиксели контуров, для сравнения которых применяются карты расстояний. Однако такой подход может приводить к ложным сопоставлениям в зонах пересечения контуров и многократным сравнениям одной и той же точки контура, и, как следствие, - к ошибке в оценке видимости. Поэтому для решения задачи определения видимости вулканов авторами предложен метод представления контуров с помощью параметрической модели, в виде ломаных линий с точками ветвления в вершинах [15]. На основе дис- кретной карты границ, полученной с помощью детектора Canny [16] выделяются последовательности пикселей между точками ветвлений и концами контуров (рис. $1, a)$. Для этого строится граф, в котором пиксели границ являются вершинами, а пиксели, имеющие общую сторону или угол, соединяются рёбрами, аппроксимирующими исходные границы по рекурсивному алгоритму (рис. 1, б). Пример построенных параметрических контуров представлен на рис. 1, 8 .

На начальном этапе анализа, для каждой камеры формируется набор из предварительно отобранных вручную снимков, сделанных в хороших погодных условиях при хорошей освещенности и в разное время года. Такие снимки будем считать эталонными, их минимальное количество для эффективной работы алгоритма должно быть не менее трех. По описанной выше схеме для каждого эталонного снимка строятся параметрические контуры, затем на основе полученных наборов формируется один результирующий, составленный из контуров, входящих не менее чем в $\gamma$ снимков (рис. 2, $а$, б). Таким образом, параметр $\gamma$ позволяет контролировать вклад наиболее характерных контуров рассматриваемого вулкана для получения наиболее точных оценок при анализе исследуемых снимков.

Набор эталонных контуров дифференцируется на внешние и внутренние. Такое разделение позволяет правильно анализировать снимки, сделанные, например, в условиях солнечной засветки, когда виден лишь общий контур вулкана (рис. 3, a). В данном случае анализ по общему набору контуров приведет к заниженной оценке видимости, в то время как внешние контуры вулкана будут полностью видны. Внешние контуры составляются из участков контуров, имеющих максимальную ординату точки пересечения с вертикальными линиями, нанесенными на изображение с расстоянием в один пиксель (рис. 3, б). Остальные контуры относятся к внутренним.

На этапе анализа снимка параметрические контуры сравниваются с эталонными. Ввиду воздействия на камеру факторов внешней среды (ветровая нагрузка, температурные вариации и т. п.) контуры на последовательно полученных снимках могут иметь смещение друг относительно друга. Для его компенсации применяется авторский алгоритм [15], основанный на методе сравнения дискретных контуров с помощью карты расстояний [17].

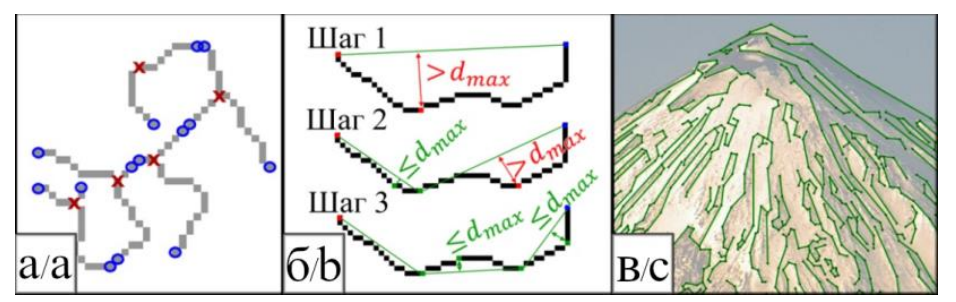

Pис. 1. Построение параметрических контуров: а) точки пересечения (красные кресты) и конечные точки (синие окружности); б) рекурсивное построение линий контуров; в) пример построенных контуров для снимка вулкана Ключевской

Fig. 1. Building parametric contours: a) intersection points (red crosses) and end points (blue circles); b) recursive edges build; c) example contours for Klyuchevskoy volcano image 


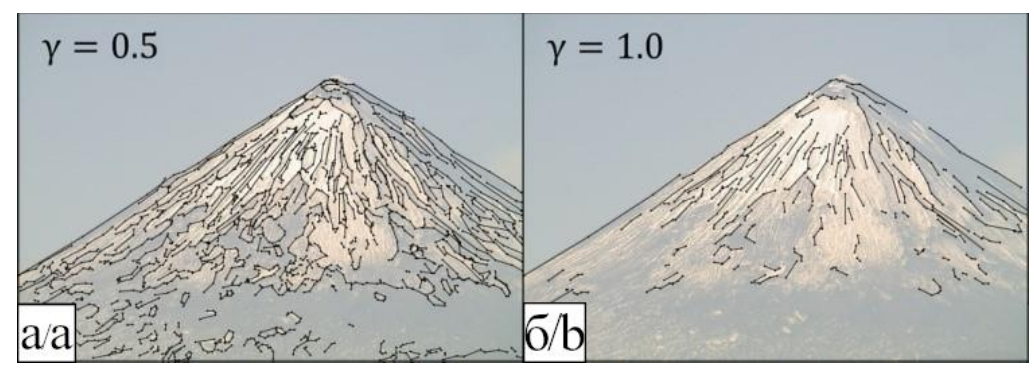

Pис. 2. Примеры эталонных контуров для разных значений $\gamma$

Fig. 2. Reference contours example for different $\gamma$

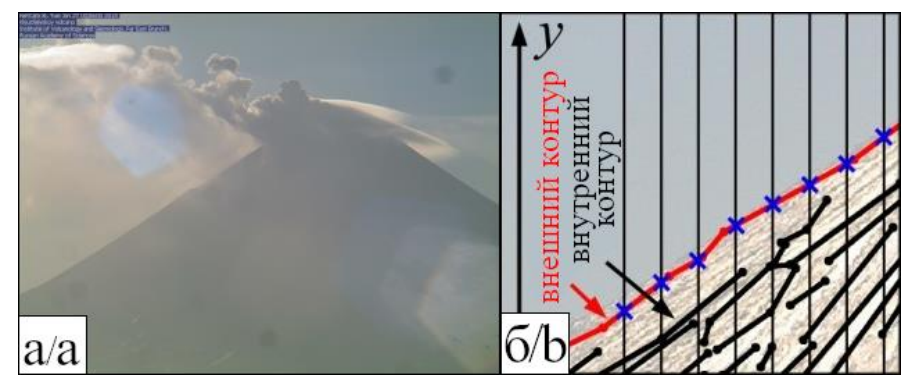

Рис. 3. Анализ контуров: а) пример снимка с плохой видимостью внутренних контуров; б) разделение конутров на внешние и внутренние (вертикальные линии прорежены)

Fig. 3. Contour analysis: a) images example with low internal contours visibility; b) extraction of internal and external contours (with vertical lines thinning)

Итоговая оценка видимости контуров вулкана на снимке $\sigma$ определяется выражением:

$$
\sigma=\max \left(\begin{array}{c}
\min \left(1, \frac{\sigma_{\text {ext }}^{\prime}}{\min _{i=1,2, \ldots, m} \bar{\sigma}_{\text {ext }, i}}\right), \\
\min \left(1, \frac{\sigma_{\text {int }}^{\prime}}{\min _{i=1,2, \ldots, m} \bar{\sigma}_{\text {int }, i}}\right)
\end{array}\right),
$$

где $\sigma_{\text {ext }}^{\prime}$ и $\sigma_{\text {int }}^{\prime}-$ оценки, полученные при сравнении контуров тестового изображения с внешними и внутренними эталонными контурами, а $\bar{\sigma}_{\text {ext }, i}$ и $\bar{\sigma}_{\text {int }, i}-$ оценки, полученные при сравнении контуров $i$-го эталонного изображения с внешними и внутренними эталонными контурами, $m$ - количество эталонных снимков.

На некоторых снимках, несмотря на частичную облачность, можно различить основные контуры вулкана и увидеть возможные признаки его активности. При этом оценка $\sigma$ для данного снимка может быть занижена ввиду плохой видимости основных эталонных контуров. Для указанных случаев оценка видимости контуров $\sigma$ корректируется с помощью оценки частотной характеристики изображения $\rho \in[0,1]$, которая вычисляется на основе вектора вклада октав частот в формирование яркостной компоненты изображения [15]. Аналогично подходу для контуров частотные характеристики вычисляются для эталонных изображений и затем сравниваются с соответствующими характеристиками анализируемых изображений. На рис. 4 представлены примеры вычисленных частотных характеристик для снимков вулкана Ключевской, сделанных при разных погодных условиях и результаты их сравнения с эталонными характеристиками.

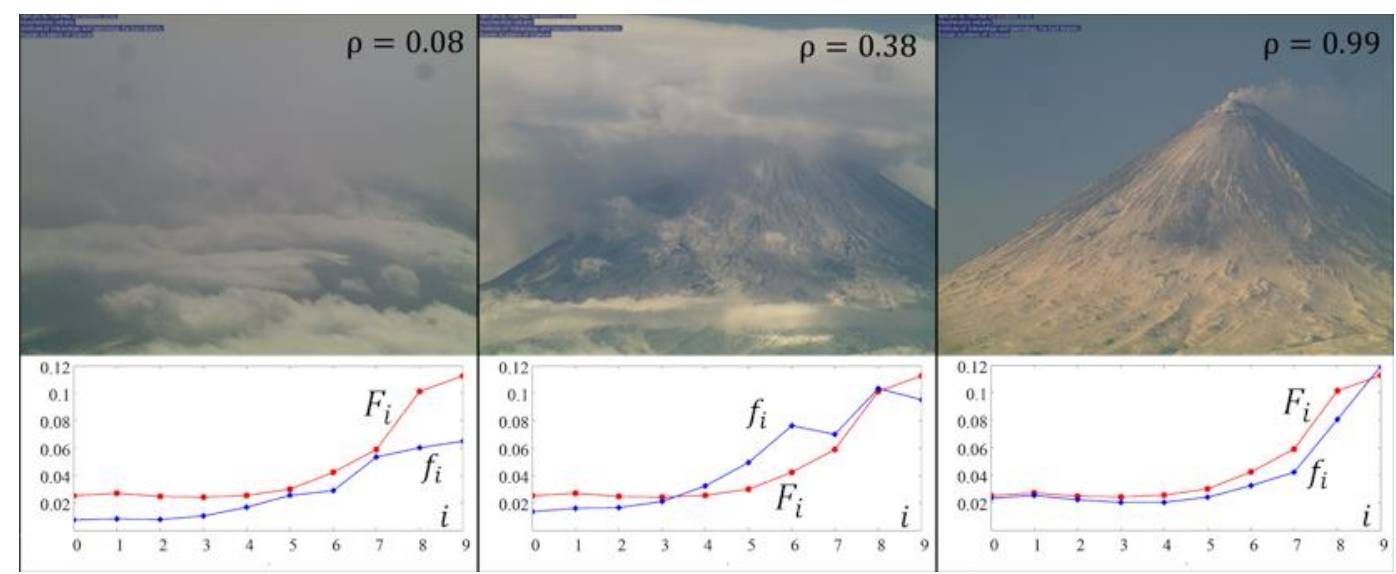

Pис. 4. Примеры сравнения частотных характеристик для снимков вулкана Ключевской, сделанных при разных погодных условиях; $F$ - вектор вклада октав частот для эталонного набора снимков, $f$ - для анализируемого снимка

Fig. 4. Examples of frequency characteristics comparison for Klyuchevskoy volcano images taken in different weather; $F-$ octave frequency contribution vector for reference images, $f$-for the analyzed image 
Корректировка оценки выполняется для снимков, оценка $\sigma$ для которых находится в $\Delta$-окрестности заданного порога $\tau$, итоговая оценка видимости определяется по формуле:

$$
\alpha=\sigma \mathrm{f}(\sigma)+\rho(1-\mathrm{f}(\sigma))
$$

где

$$
f(\sigma)=\min \left(1, \frac{1}{\Delta^{2}}(\sigma-\tau)^{2}\right)
$$

Описанный выше комплексный алгоритм определения видимости был протестирован на выборке снимков вулканов Шивелуч, Ключевской и Кизимен объемом 3000 штук на каждый объект. Для вулканов Шивелуч и Ключевской в 1 \% случаев итоговая оценка была занижена или завышена, для вулкана Кизимен - в 2 \% случаев [15]. Тестирование показало, что на ошибку в оценке влияют, прежде всего, особенности настроек камеры, такие как разрешение (чем оно выше, тем больше характеристических контуров можно различить на снимке), а также доля изображения наблюдаемого вулкана от общей площади снимка («зум» камеры).

\section{Алгоритм обнаружения яркостных аномалий}

Предложенный выше алгоритм вычисления оценки видимости вулкана на основе анализа видимости его контуров применим лишь для дневных снимков. Для изображений, полученных в ночное время с применением камер, снимающих в видимом и ближнем инфракрасном диапазонах разработан алгоритм, основанный на поиске и выделении областей с яркостными аномалиями. Под аномалией понимается часть снимка, яркость которой превышает яркость окрестности и спадает от центра к краям (рис. 5). Как правило, такие аномалии соответствуют возможным признакам активности вулкана (например, излияниям лавы из кратера).

Подход, основанный на выделении контуров на изображении, трудно применим для поиска яркостных аномалий из-за наличия шума. Кроме того, геометрическая форма аномалий сильно отличается от снимка к снимку и не подлежит сравнению с выбран- ным эталоном, а также может быть похожей на другие яркие пятна.

Суть предложенного алгоритма заключается в определении центров аномалий с помощью многомасштабного DoG (Difference of Gaussian) детектора [18] путем нахождения максимумов на DoG-слоях. Центру каждой аномалии ставится в соответствие вектор признаков. В качестве таких признаков рассматриваются: значение DoG-функции в центре, вытянутость аномалии, отношение периметра к минимально возможному периметру (сложность границы), асимметричность значений на краях, отношение перепада яркости центр-основание к значению яркости в центре, само значение яркости в центре и номер масштабного слоя, на котором найдена данная аномалия. Найденные признаки используются в качестве входных данных для SVM-классификатора [19] на основе радиальной базисной функции [20], который определяет принадлежность найденных признаков к условным классам - «яркостная аномалия» (свидетельствующая о возможной активности вулкана) и «ложная аномалия».

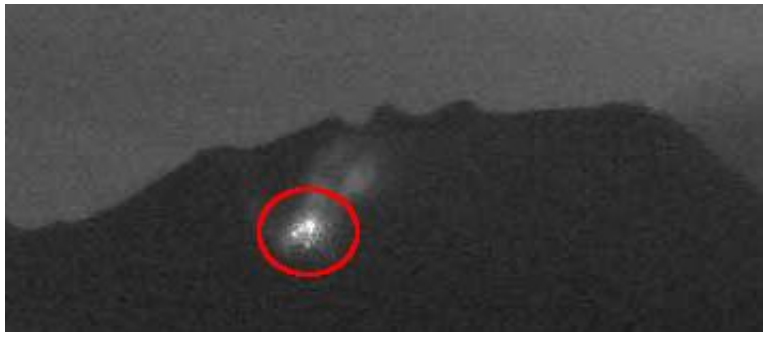

Puc. 5. Пример яркостной аномалии на ночном снимке вулкана

Fig. 5. Brightness anomaly example for volcano night image

Апробация алгоритма обнаружения яркостных аномалий проведена на ночных снимках вулкана Шивелуч. На размеченной выборке из 5068 снимков классификатор показал ложный результат в 2 \% случаев. На рис. 6 показан пример классифицированных аномалий.

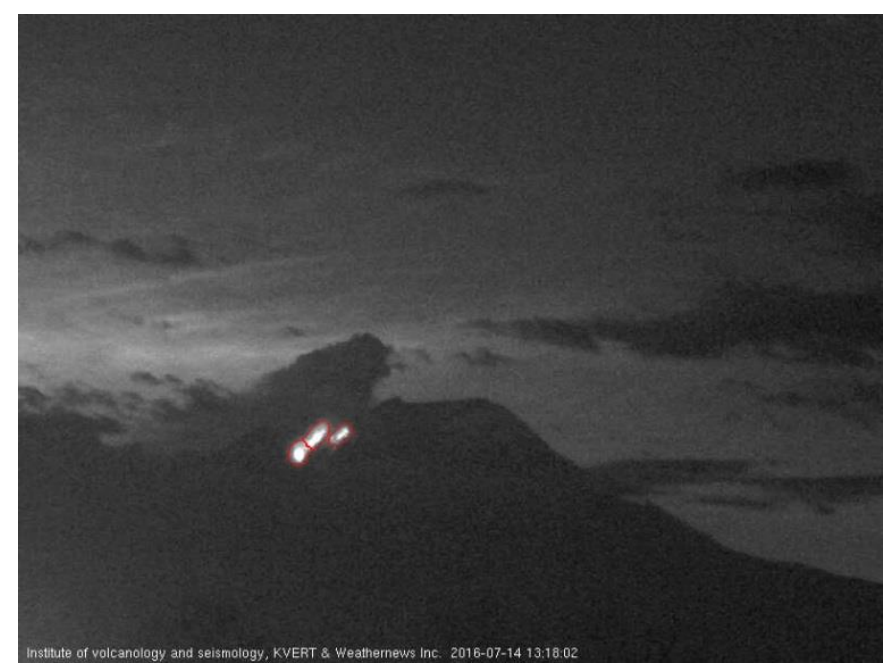

Рис. 6. Яркостные аномалии, найденные на ночном снимке вулкана Шивелуч

Fig. 6. Brightness anomalies found for Sheveluch night image 


\section{Система автоматизированного анализа} фотоснимков вулканов

Разработанные алгоритмы были реализованы в виде программного кода на языке $\mathrm{C}++$ и внедрены в работу автоматизированной информационной системы «Сигнал» [21], обеспечивающей работу сети видеонаблюдения за вулканами Камчатки [3]. При поступлении очередного снимка в архив формируется и помещается в очередь соответствующая задача (рис. 7). Диспетчер очереди Gearman передает параметры задания обработчику, который производит соответствующий анализ снимка. Полученные peзультаты записываются в базу данных и доступны для работы посредством созданных пользовательских web-интерфейсов.

Система имеет два основных режима работы: оперативный, предусматривающий автоматическую фильтрацию изображений по установленным заранее значениям (по умолчанию, при значении $\sigma>0,7$ ), и экспертный - позволяющий просматривать снимки с заданной степенью видимости вулкана, а также отмечать их в случае заниженной или завышенной оценки изображения (рис. 8). Отдельно можно работать с наборами снимков, на которых в автоматическом режиме были зафиксированы яркостные аномалии и отмечать ложноположительные результаты (рис. 9).

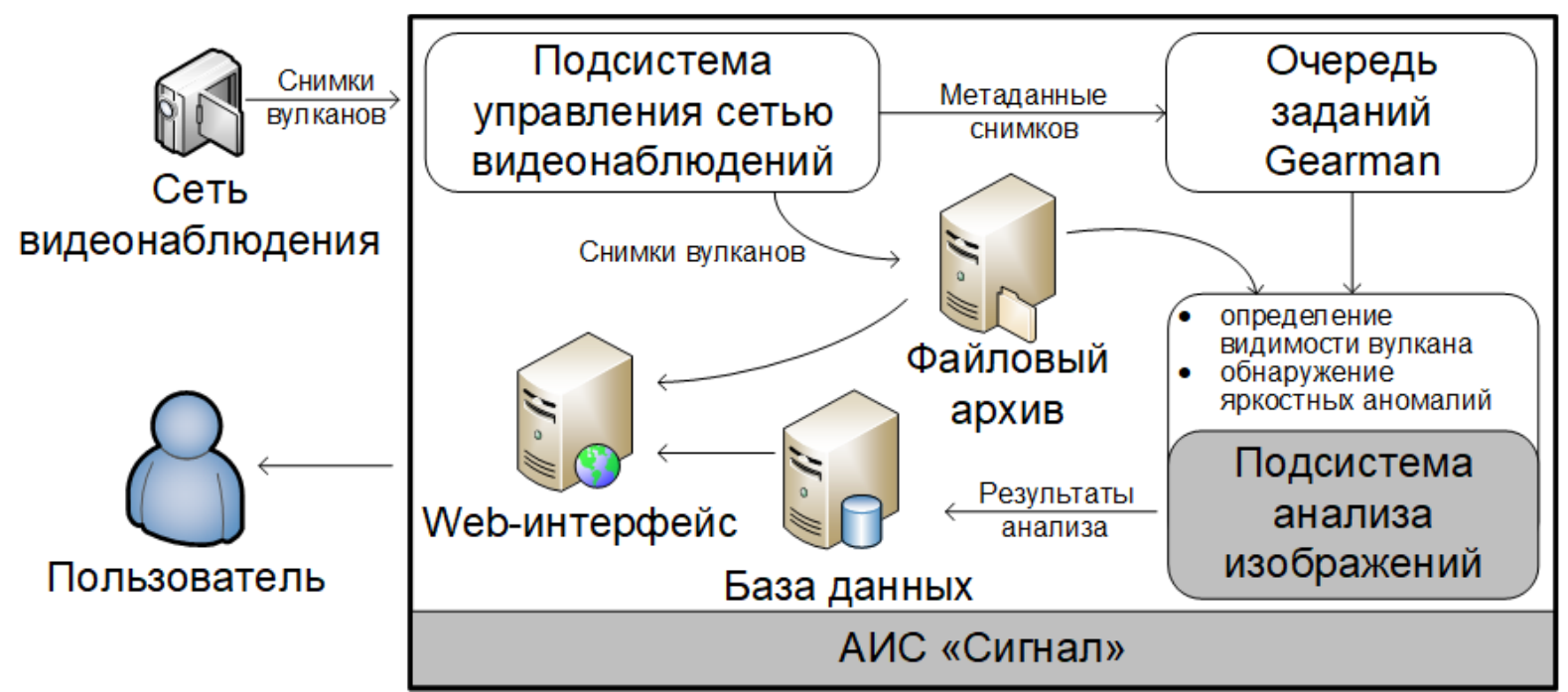

Рис. 7. Архитектура компьютерной системы для анализа изображений вулканов

Fig. 7. Volcano image analyze system architecture

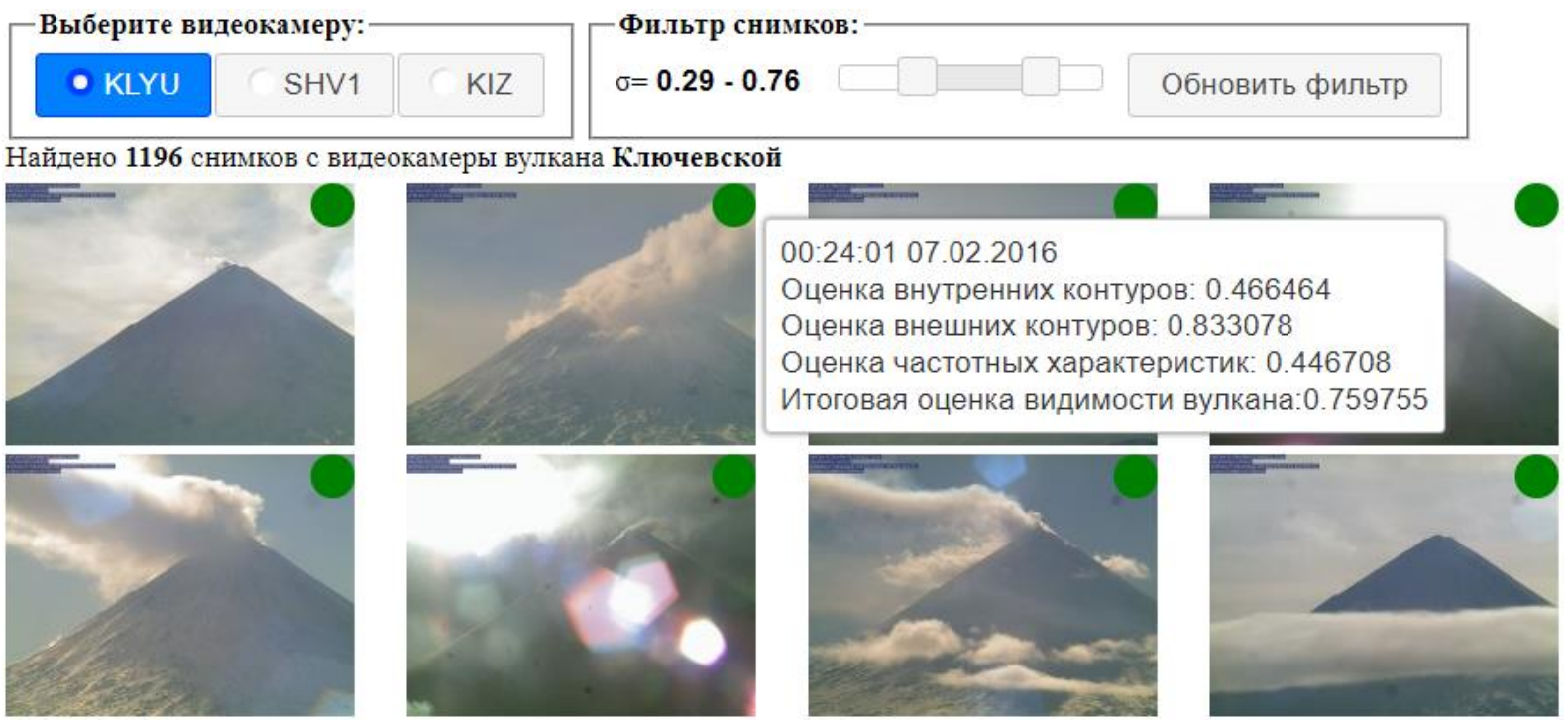

Pис. 8. Экспертный режим работы с каталогом снимков, классифицированных по степени видимости вулкана

Fig. 8. Expert user interface to browse images classified by volcano visibility 
Известия Томского политехнического университета. Инжиниринг георесурсов. 2020. Т. 331. № 3. 52-60

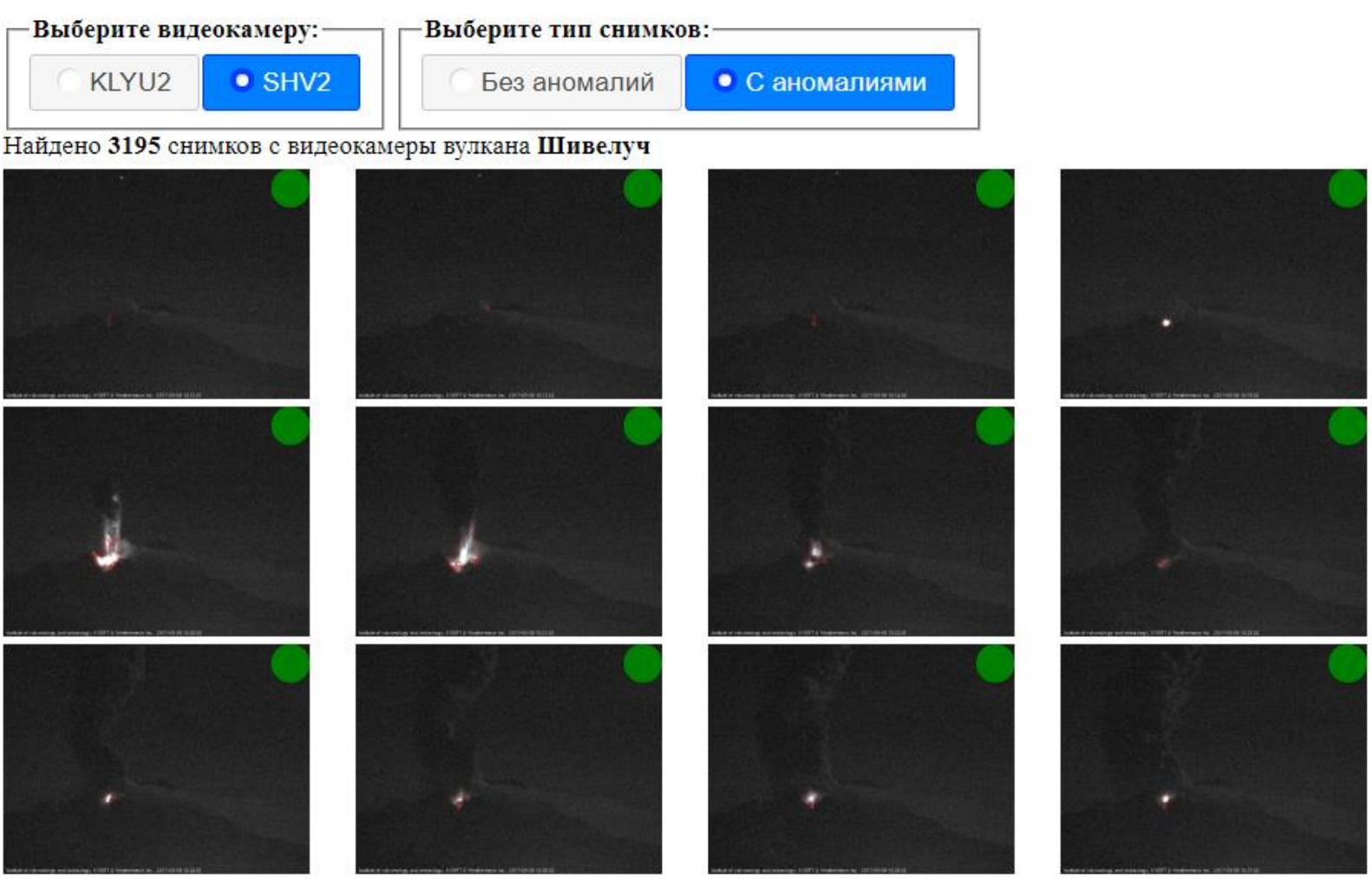

Pис. 9. Интерфейс просмотра снимков с найденными яркостными аномалиями (приведены снимки для эксплозивноэкструзивного извержения вулкана Шивелуч 22:01 07.09.2017 UTC)

Fig. 9. User interface to browse images with brightness anomalies found (images for explosive-extrusive eruption of Sheveluch volcano 22:01 07.09.2017 UTC)

\section{Заключение}

В рамках проведенных исследований были разработаны алгоритмы для анализа изображений вулканов, которые позволяют эффективно решать задачи оценки видимости вулкана и поиска яркостных аномалий. На их основе созданы и апробированы компьютерные средства для фильтрации изображений вулканов и организации работы архива системы видеонаблюдения за вулканами Камчатки.

Исследования проводились при частичной финансовой поддержке гранта РФФИ № 20-37-70008. При обработке данных были использованы вычислительные технологии, разработанные в рамках гранта РФФИ № 18-29-03100.

\section{СПИСОК ЛИТЕРАТУРЫ}

1. Sorokin A.A., Makogonov S.V., Korolev S.P. The information infrastructure for collective scientific work in the Far East of Russia // Scientific and Technical Information Processing. 2017. - V. 44. - № 4. - P. 302-304.

2. Камчатский филиал Федерального исследовательского центра «Единая геофизическая служба PAH». URL: http://www.emsd.ru (дата обращения: 26.12.2019).

3. The Kamchatka volcano video monitoring system / A. Sorokin S. Korolev, I. Romanova, O. Girina, I. Urmanov // Proc. of 2016 6th International Workshop on Computer Science and Engineering; WCSE 2016. - Tokyo, Japan, 17-19 June 2016. - P. 734-737.

4. Volcanic eruption monitoring by thermal image correlation: Pixe offsets show episodic dome growth of the Colima volcano / T. Walter, D. Legrand, H.D. Granados, G. Reyes, R. Arambula // Journal of Geophysical Research: Solid Earth. - 2013. - V. 118. P. 1408-1419. - DOI: 10.1002/jgrb.50066.
При проведении численных расчетов использовано оборудование Центра коллективного пользования «Центр данных ДВО РАН» (ВЦ ДВО РАН, г. Хабаровск) [1] и Федерального исследовательского иентра «Информатика и управление РАН» (г. Москва).

В качестве исходных данных в работе использовался архив системы видеонаблюдения за вулканами Камчатки, развиваемой при частичной поддержке Комплексной программы фундаментальных научных исследований ДВО РАН (проект № 18-5-091), а также снимки вулкана Кизимен, доступные на сайте Камчатского филиала Федерального исследовательского иентра «Единая геофизическая служба РАН» [2].

5. Patrick M., Swanson D., Orr T. Automated tracking of lava lake level using thermal images at Kilauea Volcano, Hawaii // Journal of Applied Volcanology. - 2016. - V. 5. - № 6. - P. 1-7. DOI: 10.1186/s13617-016-0047-0

6. Ando B., Pecora E. An advanced video-based system for monitoring active volcanoes // Computers \& Geosciences. - 2006. - V. 32. № 1. - P. 85-91. DOI: 10.1016/j.cageo.2005.05.004

7. Walter T.R. Low cost volcano deformation monitoring: optical strain measurement and application to Mount St. Helens data // Geophysical Journal International. - 2011. - V. 186. - № 2. P. 699-705. DOI: 10.1111/j.1365-246X.2011.05051.x.

8. Using computer vision techniques to generate embedded systems for monitoring volcanoes in Ecuador with trajectory determination / F. Viteri, K. Barrera, C. Cruz, D. Mendoza // Journal of Engineering and Applied Sciences. - 2018. - V. 13. - № 3 SI. P. 3164-3168. DOI: 10.3923/jeasci.2018.3164.3168.

9. Rabal H.J., Braga J.R.A. Dynamic laser speckle and applications. - Boca Raton: CRC Press, 2009. - 282 p. 
10. Olivo-Marin J.C. Extraction of spots in biological images using multiscale products // Pattern Recognition. - 2002. - V. 35. № 9. - P. 1989-1996.

11. Sutter Th., Nater F., Sigg Ch. Camera based visibility estimation // Proc. TECO-2016. Technical Conference on Meteorological and Environmental Instruments and Methods of Observation. - Madrid, Spain, 27-30 September 2016. - V. 2 (64). - P. 1-9.

12. Caraffa L., Tarel J.-Ph. Daytime fog detection and density estimation with entropy minimization // ISPRS Annals of the Photogrammetry, Remote Sensing and Spatial Information Sciences. - 2014. - V. II. - № 3. - P. 25-31.

13. Image change detection algorithms: a systematic survey / R.J. Radke, S. Andra, O. Al-Kofahi, B. Roysam // IEEE Transactions on Image Processing. - 2005. - V. 14. - № 3. P. 294-307.

14. Comparative study of background subtraction algorithms / Y. Benezeth, P.-M. Jodoin, B. Emile, H. Laurent, Ch. Rosenberger // Journal of Electronic Imaging, Society of Photo-optical Instrumentation Engineers. - 2010. - V. 19. - № 3. - P. 033003. DOI: 10.1117/1.3456695.

15. Анализ изображений для определения видимости вулканов / А.Н. Камаев, И.П. Урманов, А.А. Сорокин, Д.А. Карманов, С.П. Королёв // Компьютерная оптика. - 2018. - Т. 42. - № 1. C. 128-140. DOI: $10.18287 / 2412-6179-2018-42-1-128-140$.
16. Canny J. A computational approach to edge detection. Pattern analysis and machine intelligence // IEEE Transactions on PAMI. - November 1986. - V. 8. - № 6. - P. 679-698.

17. Borgefors G. Distance transformations in digital images // CVGIP. - June 1986. - V. 34. - № 3. - P. 344-371.

18. Lowe D.G. Distinctive image features from scale-invariant keypoints // International Journal of Computer Vision. - 2004. V. 60. - № 2. - P. 91-110.

19. Shmilovici A. Support vector machines // Data Mining and Knowledge Discovery Handbook. - Boston, MA, USA: Springer, 2009. - P. 257-276.

20. Vert J., Tsuda K., Schölkopf B. A primer on kernel methods // Kernel methods in computational biology. - Cambridge, MA: MIT Press, 2004. - V. 47. - P. 35-70.

21. Автоматизированная информационная система «Сигнал»: исследование и оперативный мониторинг опасных природных явлений в Дальневосточном регионе / А.А. Сорокин, С.П. Королев, С.И. Мальковский // Современные проблемы дистанционного зондирования Земли из космоса. - 2019. - Т. 16 . № 3. - C. 238-248. DOI: 10.21046/2070-7401-2019-16-3-238-248.

Поступила 12.01.2020 2.

\section{Информация об авторах}

Урманов И.П., научный сотрудник Вычислительного центра Дальневосточного отделения Российской академии наук.

Сорокин A.A., кандидат технических наук, главный научный сотрудник Вычислительного центра Дальневосточного отделения Российской академии наук.

Королев С.П., научный сотрудник Вычислительного центра Дальневосточного отделения Российской академии наук.

Мальковский С.И., научный сотрудник Вычислительного центра Дальневосточного отделения Российской академии наук. 
UDC 004.932 .2

\title{
DEVELOPING VOLCANO IMAGE ANALYSIS ALGORITHMS TO MONITOR DANGEROUS NATURAL PHENOMENA
}

Igor P. Urmanov'1, urmanov@ccfebras.ru

\section{Aleksei A. Sorokin',} alsor@febras.net

Sergey P. Korolev', serejk@febras.net

\author{
Sergey I. Malkovsky ${ }^{1}$, \\ sergey.malkovsky@gmail.com \\ 1 Computing Center FEB RAS, \\ 65, Kim-Yu-Chen street, Khabarovsk, 680000, Russia.
}

Relevance. Video surveillance systems are the most important type of instrumental means of observing the state of volcanoes. The high intensity of data collection contributes to the formation of a huge array of images. At the same time climatic peculiarities of the investigated objects location as well as not always stable operation of data transmission channels cause a large number of spoiled or uninformative images. Additional time is spent on viewing them and excluding them from the archive by specialists, which, in general, may affect the speed of decision-making, related to informing the interested parties about the occurrence of natural hazards. In this regard, the task of developing methods and technologies of image analysis and filtering for creating an effective system of video surveillance of volcanoes is relevant.

The aim of the research is to develop the algorithms for image analysis of volcanoes.

Methods: Canny boundary detector, discrete contours transformation into parametric ones in the form of broken lines, calculation of reference characteristics and comparison of the obtained data with them, analysis of frequency characteristics of the image using Haar transformation, decomposition of the image into a multiscale pyramid, drawing up a vector of characteristic visual features of an object, detection of the object being searched for using an SVM-classifier.

Results: algorithms of photo analysis to assess the visibility of volcanoes and search for brightness anomalies.

Conclusion. The considered study resulted in developing volcano image analysis algorithms. The methods and approaches used make it possible to effectively solve the problem of assessing the visibility of an object and searching for brightness anomalies. The system capabilities provide a sufficient set of tools to effectively solve the problem of image filtering and searching for brightness anomalies as a possible sign of a volcano's activity. The results obtained can be used in volcano activity monitoring systems to ensure aviation safety and population protection.

\section{Key words:}

Volcano images, volcano activity, brightness anomaly, algorithm, machine learning, information system.

The reported study was partially funded by RFBR according to the research project № 20-37-70008». Computational technologies were used in data processing, developed in the framework of the RFBR grant no. 18-29-03100. The equipment of the Shared Facility Center «Data Center of FEB RAS» (Computing Center FEB RAS, Khabarovsk) [1] and the Federal Research Center "Informatics and Control RAS» (Moscow) were used for numerical calculations.

The archive of the video monitoring system for Kamchatka volcanoes and images of the Kizimen volcano available on the website of the Kamchatka branch of the Federal Research Center "Geophysical Survey RAS» [2] were used as initial data in the work. The system was developed with partial support of the Complex Program for Basic Research of the Far East Branch of the Russian Academy of Sciences (project no. 18-5-091).

\section{REFERENCES}

1. Sorokin A.A., Makogonov S.V., Korolev S.P. The Information infrastructure for collective scientific work in the Far East of Russia. Scientific and Technical Information Processing, 2017, vol. 44, no. 4, pp. 302-304.

2. Kamchatsky filial federalnogo issledovatelskogo tsentra «Edinaya geofizicheskaya sluzhba RAN» [Kamchatka branch of the Federal Research Center «Geophysical Survey RAS». Available at: http://www.emsd.ru (accessed 26 December 2019).

3. Sorokin A., Korolev S., Romanova I., Girina O., Urmanov I. The Kamchatka volcano video monitoring system. Proc. of $20166^{\text {th }}$ International Workshop on Computer Science and Engineering; WCSE 2016. Tokyo, Japan, 17-19 June 2016. pp. 734-737.

4. Walter T., Legrand D., Granados H.D., Reyes G., Arambula R. Volcanic eruption monitoring by thermal image correlation: Pixel offsets show episodic dome growth of the Colima volcano. Journal of Geophysical Research: Solid Earth, 2013, vol. 118, pp. 14081419. DOI: $10.1002 /$ jgrb.50066.

5. Patrick M., Swanson D., Orr T. Automated tracking of lava lake level using thermal images at Kîlauea Volcano, Hawaii. Journal of Applied Volcanology, 2016, vol. 5, no. 6, pp. 1-7. DOI: 10.1186/s13617-016-0047-0

6. Ando B., Pecora E. An advanced video-based system for monitoring active volcanoes. Computers \& Geosciences, 2006, vol. 32, no. 1, pp. 85-91. DOI: 10.1016/j.cageo.2005.05.004

7. Walter T.R. Low cost volcano deformation monitoring: optical strain measurement and application to Mount St. Helens data. Geophysical Journal International, 2011, vol. 186, no. 2, pp. 699-705. DOI: 10.1111/j.1365-246X.2011.05051.x. 
8. Viteri F., Barrera K., Cruz C., Mendoza D. Using computer vision techniques to generate embedded systems for monitoring volcanoes in Ecuador with trajectory determination. Journal of Engineering and Applied Sciences, 2018, vol. 13, no. 3 SI, pp. 3164-3168. DOI: 10.3923/jeasci.2018.3164.3168.

9. Rabal H.J., Braga J.R.A. Dynamic laser speckle and applications. Boca Raton, CRC Press, 2009. $282 \mathrm{p}$.

10. Olivo-Marin J.C. Extraction of spots in biological images using multiscale products. Pattern Recognition, 2002, vol. 35, no. 9, pp. 1989-1996.

11. Sutter Th., Nater F., Sigg Ch. Camera based visibility estimation. Proc. TECO-2016. Technical Conference on Meteorological and Environmental Instruments and Methods of Observation. Madrid, Spain, 27-30 September 2016. Vol. 2 (64), pp. 1-9.

12. Caraffa L., Tarel J.-Ph. Daytime Fog Detection and Density Estimation with Entropy Minimization. ISPRS Annals of the Photogrammetry, Remote Sensing and Spatial Information Sciences, 2014, vol. II, no. 3, pp. 25-31.

13. Radke R.J., Andra S., Al-Kofahi O., Roysam B. Image change detection algorithms: a systematic survey. IEEE Transactions on Image Processing, 2005, vol. 14, no. 3, pp. 294-307.

14. Benezeth Y., Jodoin P.-M., Emile B., Laurent H., Rosenberger Ch. Comparative study of background subtraction algorithms. Journal of Electronic Imaging, Society of Photo-optical Instrumentation Engineers, 2010, vol. 19, no. 3. DOI: 10.1117/1.3456695.
15. Kamaev A.N., Urmanov I.P., Sorokin A.A., Karmanov D.A., Korolev S.P. Images analysis for automatic volcano visibility estimation. Computer Optics, 2018, vol. 42, no. 1, pp. 128-140. DOI: 10.18287/2412-6179-2018-42-1-128-140. In Rus.

16. Canny J. A computational approach to edge detection. Pattern analysis and machine intelligence. IEEE Transactions on PAMI, November 1986, vol. 8, no. 6, pp. 679-698.

17. Borgefors G. Distance transformations in digital images. CVGIP, June 1986, vol. 34, no. 3, pp. 344-371.

18. Lowe D.G. Distinctive image features from scale-invariant keypoints. International Journal of Computer Vision, 2004, vol. 60 , no. 2, pp. 91-110.

19. Shmilovici A. Support vector machines. Data Mining and Knowledge Discovery Handbook. Boston, MA, USA. Springer, 2009. pp. $257-276$.

20. Vert J., Tsuda K., Schölkopf B. A primer on kernel methods. Kernel methods in computational biology. Cambridge, MA, MIT Press, 2004. Vol. 47, pp. 35-70.

21. Sorokin A.A., Korolev S.P., Malkovsky S.I. The «Signal» automated information system: research and operational monitoring of dangerous natural phenomena in the Russian Far East. Sovremennye problemy distantsionnogo zondirovaniva Zemli iz kosmosa, 2019, vol. 16, no. 3, pp. 238-248. In Rus. DOI: 10.21046/2070-7401-2019-16-3-238-248.

Received: 12 January 2020.

Information about the authors

Igor P. Urmanov, researcher, Computing Center FEB RAS.

Aleksei A. Sorokin, Cand. Sc., chief researcher, Computing Center FEB RAS.

Sergey P. Korolev, researcher, Computing Center FEB RAS.

Sergey I. Malkovsky, researcher, Computing Center FEB RAS. 